\title{
Estimation and Visualization of Sagittal Kinematics of Lower Limbs Orientation Using Body-Fixed Sensors
}

\author{
Hooman Dejnabadi*, Brigitte M. Jolles, Emilio Casanova, Pascal Fua, Member, IEEE, and \\ Kamiar Aminian, Member, IEEE
}

\begin{abstract}
A new method of estimating lower limbs orientations using a combination of accelerometers and gyroscopes is presented. The model is based on estimating the accelerations of ankle and knee joints by placing virtual sensors at the centers of rotation. The proposed technique considers human locomotion and biomechanical constraints, and provides a solution to fusing the data of gyroscopes and accelerometers that yields stable and drift-free estimates of segment orientation. The method was validated by measuring lower limb motions of eight subjects, walking at three different speeds, and comparing the results with a reference motion measurement system. The results are very close to those of the reference system presenting very small errors (Shank: $r m s=1.0$, Thigh: $r m s=1.6^{\circ}$ ) and excellent correlation coefficients (Shank: $r=0.999$, Thigh: $r=0.998$ ). Technically, the proposed ambulatory system is portable, easily mountable, and can be used for long term monitoring without hindrance to natural activities. Finally, a gait analysis tool was designed to visualize the motion data as synthetic skeletons performing the same actions as the subjects.
\end{abstract}

Index Terms-Accelerometer and gyroscope, ambulatory system, gait analysis, visualization.

\section{INTRODUCTION}

H UMAN motion capture and its visualization are usually performed based on camera, magnetic and ultrasound systems [1], [2]. Although these standard technologies allow a complete three-dimensional (3-D) kinematics of body segment they require a dedicated laboratory where the subjects should walk in a predefined specific path, and assume that data measured from only a few seconds are representative of usual performance. This constraint beside the time needed for the analysis and also the cost of these technologies has limited the use of these standard technologies in clinical practice. Ambulatory monitoring of body movement takes a different approach: collecting data

Manuscript received July 19, 2005; revised January 6, 2006. This work was supported in part by the Swiss National Foundation under Grants FNRS 3200064951 and 3200B0-105880. Asterisk indicates corresponding author.

${ }^{*}$ H. Dejnabadi is with the Ecole Polytechnique Fédérale de Lausanne (EPFL), Laboratory of Movement Analysis and Measurement, 1015 Lausanne, Switzerland (e-mail: hooman.dejnabadi@epfl.ch).

B. M. Jolles is with the University of Lausanne (CHUV), Hôpital Orthopédique de la Suisse Romande, 1011, Lausanne, Switzerland (e-mail: brigitte.jolles-haeberli@chuv.ch).

E. Casanova and P. Fua are with the Ecole Polytechnique Fédérale de Lausanne (EPFL), Computer Vision Laboratory, 1015 Lausanne, Switzerland (e-mail: emilio.casanova@epfl.ch; pascal.fua@epfl.ch).

K. Aminian is with the Ecole Polytechnique Fédérale de Lausanne (EPFL), Laboratory of Movement Analysis and Measurement, 1015 Lausanne, Switzerland (e-mail: kamiar.aminian@epfl.ch).

Digital Object Identifier 10.1109/TBME.2006.873678 from body-fixed sensors in the natural environment of the subject. In this regard, movement analysis using body fixed inertial sensors as a complementary method has many potential in clinical field [3], [4].

While standard technologies provide directly body segment position and orientation relative to a fixed referential, the outputs of inertial sensors are rather relative angles, segment acceleration or velocity. Finding 3-D segment orientation, absolute angles and complete kinematics are a major difficulty when using body fixed inertial sensors.

Orientation angle estimation using inertial sensors, consisting of accelerometers and/or rate gyroscopes has been studied by many authors. In fact, both an accelerometer and a gyroscope can measure orientation angle of a segment. However, an accelerometer is slow in response and sensitive to linear accelerations, and a gyroscope suffers from slow drift and unknown initial inclination [5]-[7]. In order to eliminate gyroscope drift, Morris [8] identified the beginning and the end of the walking cycles, and made the angle signal at the beginning and the end of the cycle equal. Tong et al. [9] applied a low-cutoff high-pass filter on the shank and thigh inclination angle signals. Time-frequency analysis (wavelet transform) was also applied to lower limb angular velocity in order to remove the drift [10]. However, all of these methods remove the dc and low-frequency information of angles. Heyn et al. [11] showed that shank and thigh inclination could be measured with eight accelerometers and two gyroscopes fixed on two rigid metal plates. They found that using these metal plates was cumbersome. Many authors designed Kalman filters to fuse gyroscope, accelerometer, and/or magnetometer signals [5]-[7], [12]-[15]. However, the performance of the filter will considerably be reduced in measuring orientation angle of segments, like shank, with fast movements and large centripetal acceleration components [5], [7].

We have recently proposed a method to estimate uniaxial joint angles based on two sensor modules, mounted on the shank and thigh, each containing two accelerometers and one gyroscope [1]. By considering the two-dimensional (2-D) model of segments, we calculated the expected signals of virtual sensors placed at knee joint with respect to the physical sensors. The method of estimating joint angle, however, does not estimate orientations of shank and thigh segments with respect to a fixed frame.

This paper presents a new complementary method to estimate shank segment orientation in sagittal plane during walking, and subsequently calculate thigh angle by adding the two values of shank and knee angles. We provide a solution to fusing data 


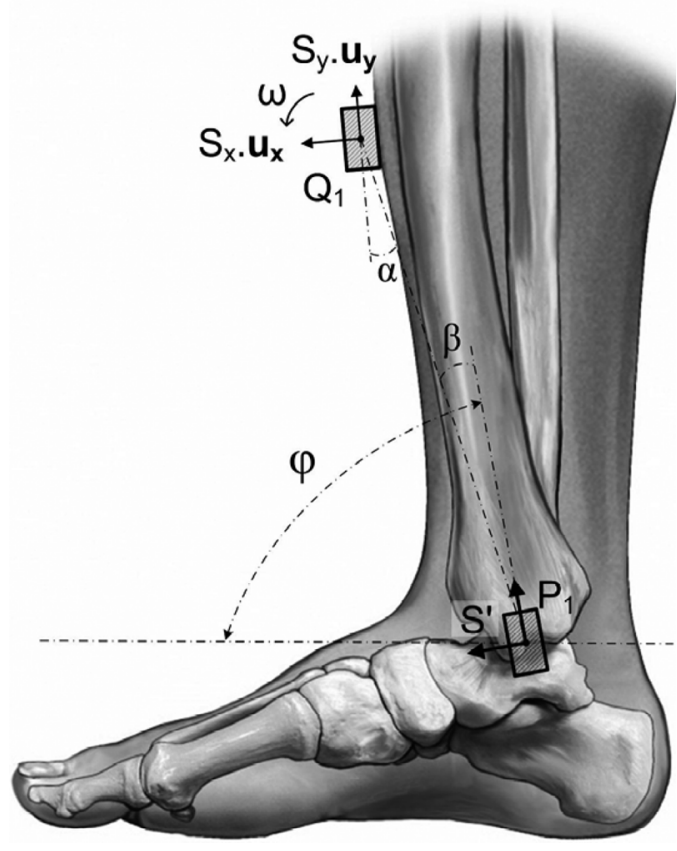

Fig. 1. Position of sensor on shank, and its corresponding virtual sensor on ankle.

from a gyroscope and a biaxial accelerometer that provides stable estimates of the segment orientation. The fusing method considers human locomotion and biomechanical constraints, and incorporates excellent dynamic response of gyroscopes and stable drift-free performance of accelerometers. A geometric calibration is needed to give position of sensors to the model. These parameters are obtained by photography. Finally, we designed a gait analysis tool to visualize the motion data as synthetic skeletons performing the same actions as the subjects.

\section{METHODS}

\section{A. Estimation of Shank Orientation}

To estimate orientation of shank segment, the kinematic data of the sensor module placed on shank at point $\mathrm{Q}_{1}$ was processed (Fig. 1). The sensor module consisted of a biaxial accelerometer and a gyroscope. Both the gyroscope and accelerometer signals contain information about the orientation of the sensor [15]. A gyroscope signal $\left(S_{g}\right)$ is the sum of angular velocity $(\omega)$ and a slowly varying offset $(b)$

$$
S_{g}=\omega+b
$$

where the offset $(b)$ is caused by electronic bias error and deviation from the sensing axis [1].

The sensor orientation $\left(\varphi_{g}\right)$ can be obtained by integration of the gyroscope signal

$$
\varphi_{g}(t)=\int S_{g} d t=\int \omega d t+d(t)
$$

where $d(t)$ including both offset and drift, distorts the sensor orientation.

On the other hand, a single-axis accelerometer measures the difference of acceleration (a) and gravity ( $(\mathrm{g})$ along its sensitive axis given by the unit vector $(\mathbf{n})$. The measured signal can, thus, be expressed as

$$
S=(\mathbf{a}-\mathbf{g}) \cdot \mathbf{n} .
$$

Similarly, by considering a biaxial accelerometer with sensitive axes along $\mathbf{u}_{\mathbf{x}}$ and $\mathbf{u}_{\mathbf{y}}$, the two measured signals were given by

$$
\begin{aligned}
& S_{x}=(\mathbf{a}-\mathbf{g}) \mathbf{u}_{\mathbf{x}} \\
& S_{y}=(\mathbf{a}-\mathbf{g}) \mathbf{u}_{\mathbf{y}} .
\end{aligned}
$$

respectively.

The acceleration vector can be rewritten in polar form

$$
\mathbf{S}=e^{i \varphi} . S
$$

where $S$ and $\phi$ represent for modulus and argument of $\mathbf{S}$.

The gravitational component can be used to make an estimation of the inclination angle [16], [17]. The inclination is defined as the angle between the sensor axes and the horizontal plane. If the acceleration (a) is small compared to the gravity $(\mathrm{g})$, the accelerometer can be used as an inclinometer, and the inclination angle is equal to the argument $(\phi)$. The problem is of course that when the segment is accelerated, the accelerometer is not an accurate inclination sensor [6]. When the acceleration is low, the amplitude of accelerometer $(S)$ corresponds rather to the gravity constant $(g)$, this is a necessary condition to detect low acceleration intervals. However, this condition alone is not enough. Since a body segment cannot sustain a constant linear acceleration very long with no rotation; therefore, the rule for detecting low acceleration is to demand $S=g$ for a certain amount of time $T_{0}$ [6], [7], [18].

In order to fuse the data from gyroscope and accelerometer, the constraints of having low accelerations on ankle during foot flat periods of gait and also during quiet standing were applied. In foot flat periods, the entire foot comes in contact with the floor and the shank segment performs a pure rotation around ankle joint, while the ankle joint does not move. In quiet standing periods, the shank segment has no rotation or translation.

Since the magnitude of acceleration on ankle is low during foot-flat and quiet standing periods, a virtual accelerometer placed on ankle and aligned with the shank segment orientation is a good estimator of inclination during those periods. Therefore, the first step was to calculate the expected signals of a virtual sensor placed on the ankle joint at point $\mathrm{P}_{1}$, and aligned with the shank segment orientation. The relationship between the virtual sensor and the physical sensor on shank could be derived from [1] as

$$
\left[\begin{array}{l}
S_{x}^{\prime} \\
S_{y}^{\prime}
\end{array}\right]=R_{-\beta} \cdot\left(R_{\alpha} \cdot\left[\begin{array}{l}
S_{x} \\
S_{y}
\end{array}\right]+\left[\begin{array}{c}
-r \cdot \omega^{2} \\
r . \dot{\omega}
\end{array}\right]\right)
$$


where $S_{x}$ and $S_{y}$ are physical accelerometer readings; $S_{x}^{\prime}$ and $S_{y}^{\prime}$ are virtual accelerometer readings. $R_{\alpha}$ and $R_{\beta}$ are axis rotation matrices of the physical and virtual sensors in relation to the direction of vector $\mathbf{r}$ by angles $\alpha$ and $\beta$, respectively (see also Fig. 1). It is assumed that the two sensors are fixed on a rigid segment, so their distance (r) is constant, and both sensors have identical angular velocities $(\omega)$. So the Coriolis term will not appear in (6).

Similarly, the virtual acceleration vector on ankle can be rewritten in polar form

$$
\mathbf{S}^{\prime}=e^{i \varphi_{a}} \cdot S^{\prime}
$$

where $S^{\prime}$ and $\varphi_{a}$ represent for modulus and argument of $\mathbf{S}^{\prime}$.

The second step was to detect the periods when the magnitude of translational acceleration on ankle is low. This magnitude could be expressed as

$$
e(t)=\left|S^{\prime}-g\right| .
$$

Then, the two necessary conditions for detecting low acceleration periods were applied on the magnitude signal $e(t)$. The first condition was to find the periods when $e(t)$ is small. So a binary mask was defined such that it has value "1" during low acceleration periods, and " 0 " otherwise. The mask can be obtained by thresholding the magnitude signal $e(t)$. However, selection of an appropriate global threshold is difficult, and varies from subject to subject and for different walking speeds. So, instead of using a single threshold value, a hysteresis thresholding method [19] was applied on $e(t)$ to obtain the mask $M_{1}$

$$
M_{1}(t)= \begin{cases}1, & \text { if } e(t)<c_{1} \\ 0, & \text { if } e(t)>c_{2} \\ p, & \text { otherwise }\end{cases}
$$

where $c_{1}$ ("hard" threshold) and $c_{2}$ ("weak" threshold, $c_{1}<c_{2}$ ) are small constant parameters chosen heuristically. All values in the magnitude signal $e(t)$ having a value less than $c_{1}$ are immediately accepted ("secure" values). Conversely, all values greater than $c_{2}$ are immediately rejected. "Potential" samples $(p)$ with values between both thresholds are accepted if they are connected to secure samples by a path of potential samples. Hysteresis thresholding is more immune to noise than simple "hard" thresholding. It helps to ensure that a noisy interval (e.g., foot flat) is not broken into multiple fragments and preserves the connectivity of the mask.

The second condition for detecting low acceleration intervals was to select only the high state $(=1)$ periods in the mask $\left(M_{1}\right)$ that persists for at least a certain amount of time $T_{0}$. So narrow pulses shorter than $T_{0}$ in the mask were eliminated. This was performed by applying a morphological "Opening" filter [20] on $M_{1}$ with window size $T_{0}$ to obtain the mask $M_{2}$

$$
M_{2}=\operatorname{OPEN}\left(M_{1}, T_{0}\right) .
$$

The window size $\left(T_{0}\right)$ was heuristically chosen as $0.1 \mathrm{~s}$.

Based on the given conditions, the shank orientation angle, estimated by the virtual accelerometer on ankle $\left(\phi_{a}\right)$, was valid during the period where the resulting mask $\left(M_{2}\right)$ had value equal to 1 . These valid values of $\phi_{a}$ were used to correct the drift in the estimated shank angle using the gyroscope $\left(\phi_{g}\right)$

$$
d(t)= \begin{cases}\varphi_{g}-\varphi_{a}, & \text { if } M_{2}=1 \\ \text { unkown, } & \text { if } M_{2}=0\end{cases}
$$

where $d(t)$ represents for the drift signal expressed in (2).

In order to estimate the drift $d(t)$ at "unknown" times, first the signal at known times were smoothed by applying a secondorder Butterworth lowpass filter. This operation would not lose any information in the signal, because the drift was expected to have low variations in time. Then an interpolation technique based on piecewise cubic hermite interpolation was applied on the drift signal at known intervals [21]. The resulting interpolant has no overshoots or oscillations during unknown times, and preserves monotonicity in the signal.

This drift is then subtracted from the angle estimated by gyroscope to yield absolute shank angle at all times

$$
\varphi_{\text {Shank }}(t)=\varphi_{g}(t)-d(t)
$$

\section{B. Estimation of Thigh Orientation}

We proposed in [1] a method of measuring knee joint flexion-extension angle $\left(\phi_{\text {Knee }}\right)$ based on two modules of sensors placed on shank and thigh. We considered the 2-D model of segments, and calculated the expected signals of virtual sensor modules placed at knee joint with respect to the physical sensor modules. The method was validated by measuring knee flexion-extension angles during walking at different speeds. Joint motion is actually the relative motions between the articulating segments. So considering the thigh segment as an articulated rigid segment connected to the shank segment, the relative motion of thigh to shank could be expressed with knee joint motion $\left(\phi_{\text {Knee }}\right)$, and hence the orientation angle of thigh segment with respect to horizontal frame could be given by

$$
\varphi_{\text {Thigh }}=\varphi_{\text {Shank }}+\varphi_{\text {Knee }} .
$$

\section{Test Protocol}

Eight healthy subjects, who had given informed consent, participated in this experiment, 5 men and 3 women, aged between 44 and $70 \mathrm{yr}$ (mean $=58.7 \mathrm{yr})$. The volunteers performed three 30 -s flat treadmill walking trials at speeds 2,3 , and $4 \mathrm{~km} / \mathrm{h}$, wearing their sport shoes.

To capture lower limbs activities, four sensor modules, each containing two accelerometers and one gyroscope, were used. Dual axis accelerometer chips ADXL202/210 and yaw rate gyro chips ADXRS150/300 were chosen. Temperature drift rates were less than $0.1 \%$ for the gyroscopes and few $\mathrm{mg}$ for the accelerometers. The sensors (dimension: $20 \mathrm{~mm} \times 20 \mathrm{~mm} \times 10 \mathrm{~mm}$ ) were mounted on both shank and thigh segments using straps (Fig. 2). However, for validation with the reference system, only the data of left shank and thigh were used. The sensing axes were adjusted in the 


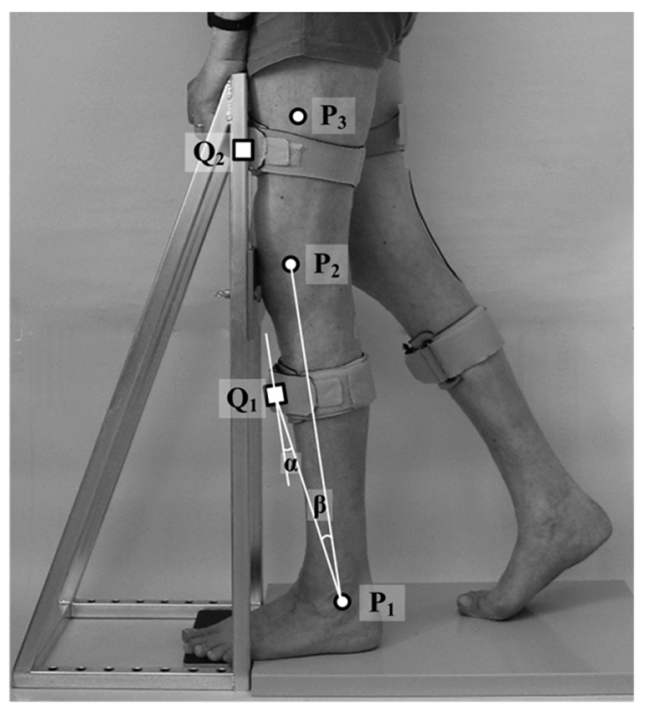

Fig. 2. Attachment of the kinematic sensors on both thighs and shanks using straps. The kinematic data are recorded by the Physilog system (dimensions: $13 \mathrm{~cm} \times 7 \mathrm{~cm} \times 3 \mathrm{~cm}$, weight: $300 \mathrm{gr}$ ) placed in a waist bag. The white circles indicate the position of markers $\left(\mathrm{P}_{1}, \mathrm{P}_{2}\right.$, and $\left.\mathrm{P}_{3}\right)$, and the white squares indicate the position of kinematic sensors $\left(\mathrm{Q}_{1}\right.$ and $\left.\mathrm{Q}_{2}\right)$.

anterio-posterior plane so that the motion in the sagittal plane could be measured. All signals were sampled at $200-\mathrm{Hz}$ using the Physilog [BioAGM, CH] ambulatory system carried on the waist [22]

A geometric calibration was required to obtain position of sensors with respect to anatomical landmarks. This information was then given to our proposed models to estimate the expected virtual sensor readings shifted to the ankle and the knee joints respectively. So before the walking trials, three small markers were pasted over the left lateral malleolus $\left(\mathrm{P}_{1}\right)$, the lateral epicondyle $\left(\mathrm{P}_{2}\right)$, and the junction of the first and second proximal lateral third of the thigh $\left(\mathrm{P}_{3}\right)$ (Fig. 2). Then, the subject stayed for a few seconds $(<5 \mathrm{~s})$ at standstill, while the system was recording kinematic parameters, a lateral view photograph was taken as well. The camera's image plane was adjusted to be in parallel with sagittal plane to avoid perspective errors. The known length of the metal frame (70 cm height) was used to calibrate the photo from pixels to metric units. This image was used to estimate the coordinates of markers and kinematic sensors. The accelerometer's readings during standstill were used to estimate the sensor's orientation with respect to horizontal plane. The calibration procedure is simple and can be completed within less than $60 \mathrm{~s}$. It is not critical to find exact positions of markers (P1, P2, and P3). For example, moving the markers 1 $\mathrm{cm}$ in each direction causes very minimal changes in the segment angles (less than $2^{\circ}$ ).

The angles $\alpha$ and $\beta$, and the length $r$ were calculated afterwards to be used in (6). The angle $\alpha$ was obtained by calculating the difference between inclination angle of the sensor $\left(\mathrm{S}_{1}\right)$ and the vector $\overrightarrow{\mathbf{P}_{\mathbf{1}} \mathbf{Q}_{\mathbf{1}}}$

$$
\alpha=\tan ^{-1}\left(\frac{\overline{S_{y_{1}}}}{\overline{S_{x_{1}}}}\right)-\tan ^{-1}\left(\frac{y_{P_{1}}-y_{Q_{1}}}{x_{P_{1}}-x_{Q_{1}}}\right)
$$
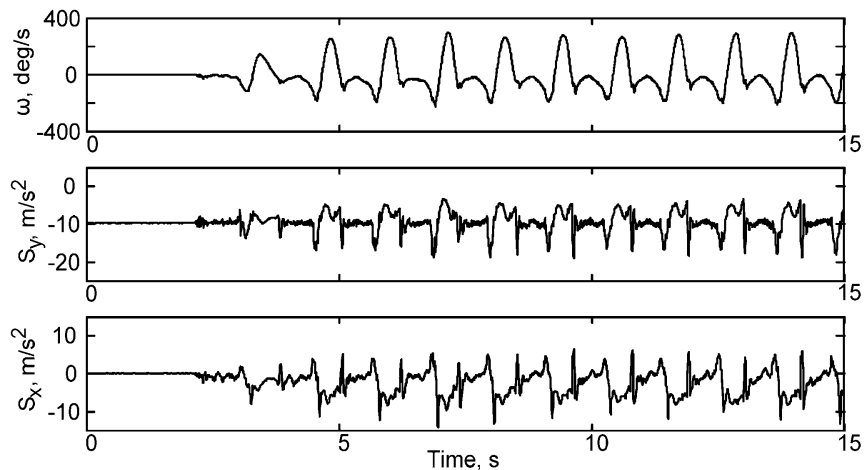

Fig. 3. Physical sensor readings on shank during walking at $3 \mathrm{~km} / \mathrm{h}$. The sensor module consists of two accelerometers $\left(\mathrm{S}_{\mathrm{x}}\right.$ and $\left.\mathrm{S}_{\mathrm{y}}\right)$ and a gyroscope $(\omega)$.

where $\overline{S_{x_{1}}}$, and $\overline{S_{y_{1}}}$ are averages of the 2-D accelerometer readings during the standstill trial.

The angle $\beta$ was obtained from the dot product formula

$$
\beta=\cos ^{-1}\left(\frac{\overrightarrow{\mathbf{P}_{\mathbf{1}} \mathbf{P}_{\mathbf{2}}} \cdot \overrightarrow{\mathbf{P}_{\mathbf{1}} \mathbf{Q}_{\mathbf{1}}}}{\left|P_{1} P_{2}\right| \cdot\left|P_{1} Q_{1}\right|}\right)
$$

where $\overrightarrow{\mathbf{P}_{\mathbf{1}} \mathbf{P}_{\mathbf{2}}}=\left[x_{P_{1}}-x_{P_{2}} \quad y_{P_{1}}-y_{P_{2}}\right]^{\prime}, \overrightarrow{\mathbf{P}_{\mathbf{1}} \mathbf{Q}_{\mathbf{1}}}=\left[x_{P_{1}}-\right.$ $\left.x_{Q_{1}} \quad y_{P_{1}}-y_{Q_{1}}\right]^{\prime}$, and finally $r=\left|P_{1} Q_{1}\right|$.

For comparison, a Zebris CMS-HS (Zebris, D) ultrasoundbased motion measurement system was used as the reference system [23]. This system consists of three fixed sonic emitters which send out a burst of ultrasound, and receivers placed on body segments. The time taken for the burst to reach each receiver is recorded. Using this delay, the distances between the receiver and each emitter can be calculated from the sound velocity. Knowing the distance from three emitters, the coordinates of the receiver placed on body segment can be computed by triangulation with an absolute accuracy better than 1.0 $\mathrm{mm}$ [24], [25] with a sampling rate of $100 \mathrm{~Hz}$. In this study, three ultrasound receivers were attached over the same adhesive markers $\left(\mathrm{P}_{1}, \mathrm{P}_{2}\right.$, and $\left.\mathrm{P}_{3}\right)$. Spatial marker positions $(x, y, z)$ were recorded and used for calculation of shank and thigh orientation angles. Synchronization between the reference and the Physilog systems was performed by electrical trigger. The angle data obtained by the body-fixed sensors were down sampled to $100 \mathrm{~Hz}$ for comparison purpose.

\section{Data Analysis}

Matlab was used for all signal processing. A third order Savitzky-Golay filter [26] was applied to smooth the accelerometers and gyroscopes signals. For comparison with the reference system, the error signal $\operatorname{Er}(t)$ was defined as the difference between the time series angle obtained by the proposed method and the reference system.

The accuracy of the results was calculated in terms of RMS, mean, and standard deviation of the error signal $(\operatorname{Er}(t))$, as 


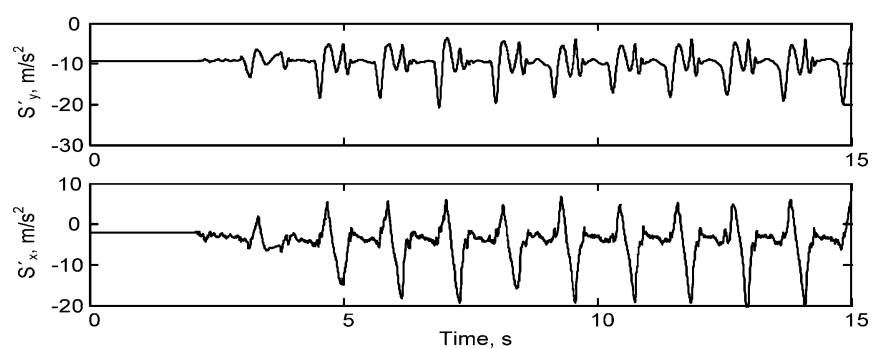

Fig. 4. Virtual accelerometer readings on ankle during walking at $3 \mathrm{~km} / \mathrm{h}$. The signals are calculated from the raw signals shown in Fig. 3.
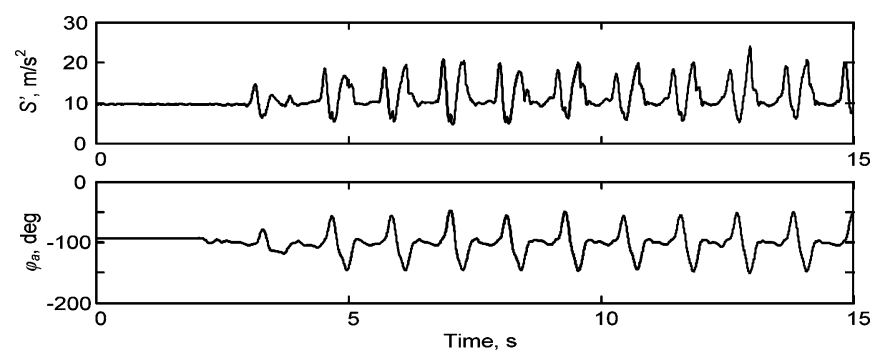

Fig. 5. Polar representation (modulus: $S^{\prime}$, argument: $\phi_{a}$ ) of the virtual accelerometer on ankle calculated from the signal shown in Fig. 4.

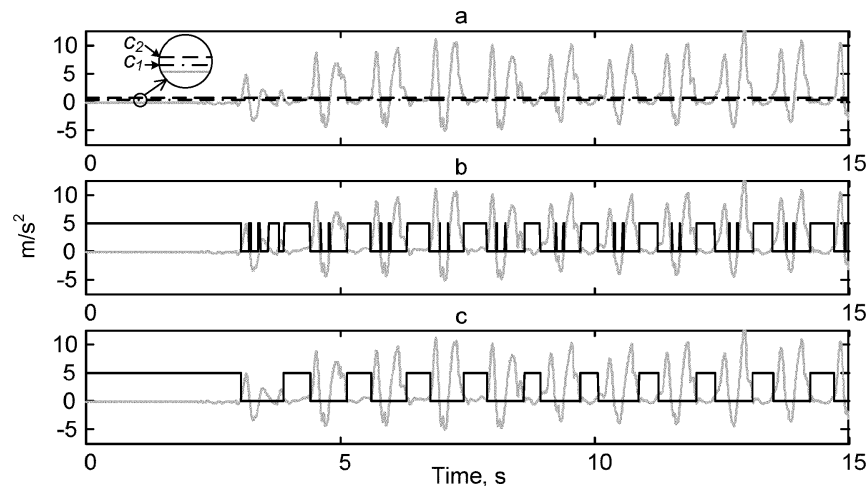

Fig. 6. Detection of the periods of motion with low acceleration by employing hysteresis thresholding and morphological filter. (a) Magnitude of translational acceleration on ankle $e(t)$ (solid gray line), and two thresholds $c_{1}$ and $c_{2}$ (dashed lines) set for hysteresis thresholding. (b) Result of hysteresis thresholding as binary mask $M_{1}$. (c) Result of morphological opening filter as binary mask $M_{2}$. The filter was applied on $M_{1}$ to eliminate narrow pulses shorter than $T_{0}=0.1 \mathrm{~s}$.

well as correlation coefficient between the orientation angles obtained by the proposed method and the reference system's data.

\section{RESULTS}

\section{A. Estimation of Shank and Thigh Orientations}

The main steps of calculating shank angle during typical walking trail at $3 \mathrm{~km} / \mathrm{h}$ are shown in Figs. 3-8. Figs. 3-5 indicate how the magnitude and phase of the virtual sensor on ankle are derived from the measured accelerometer and gyroscope signals. The measured accelerometer and gyroscope signals on shank (Fig. 3) are transformed to the virtual accelerometer reading on ankle (Fig. 4) using (5). The polar representation (modulus, argument) of the virtual accelerometer (Fig. 5) is then obtained using (6).

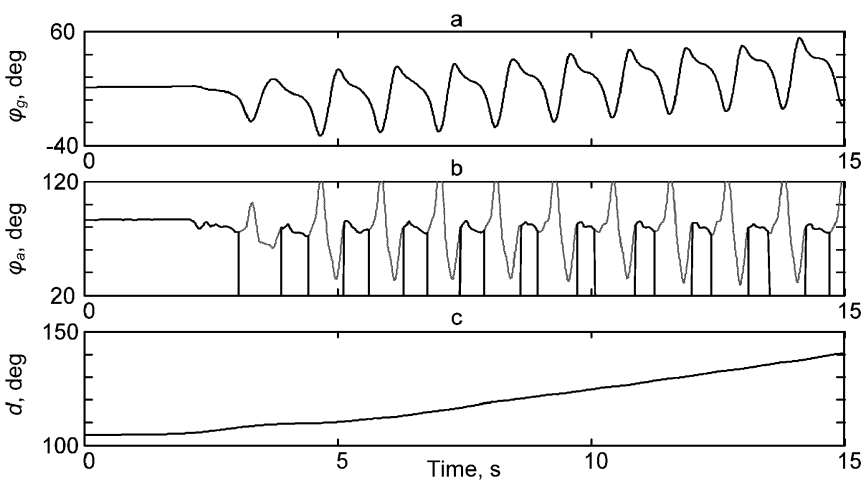

Fig. 7. Estimation of drift by combining the two angle information obtained by gyroscope and virtual accelerometer. (a) Estimated shank angle using gyroscope $\left(\phi_{g}\right)$. (b) Estimated shank angle using the virtual accelerometers $\left(\phi_{a}\right)$. The angle is valid only during low acceleration periods indicated by mask $M_{2}$. (c) Estimated drift by combining (a) and (b). The procedure consists of subtracting the two signals followed by applying piecewise cubic hermite interpolation. The estimated drift is then used to calculate the correct orientation of shank $\left(\phi_{\text {Shank }}\right)$.
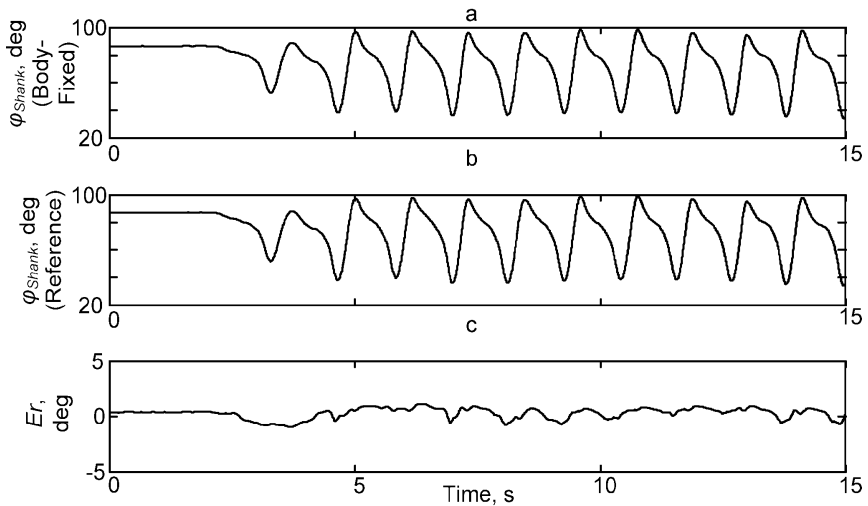

Fig. 8. Comparison between the measured angles using the proposed method and the reference angles (a) shank angle calculated by the proposed method using the body-fixed sensors. (b) Calculated from position data as measured by the reference system. (c) Difference error between the two results. Note that the scale is zoomed to -5.0 to $5.0^{\circ}$ for better viewing.
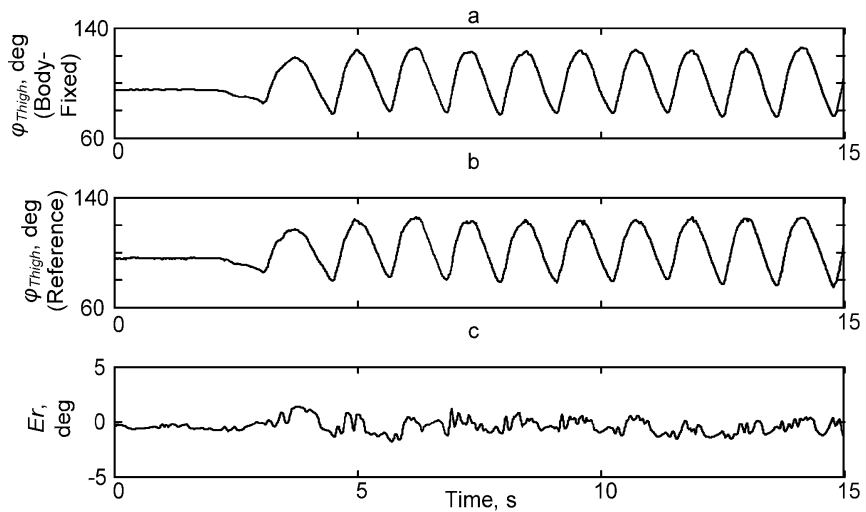

Fig. 9. Comparison between the measured angles using the proposed method and the reference angles (a) thigh angle calculated by the proposed method using the body-fixed sensors. (b) Calculated from position data as measured by the reference system. (c) Difference error between the two results. Note that the scale is zoomed to -5.0 to $5.0^{\circ}$ for better viewing.

Fig. 6 indicates how the periods of motion with low acceleration are detected by employing hysteresis thresholding and morphological filter. Fig. 6(a) shows the magnitude of translational acceleration on ankle $[e(t)$ in solid line], and two thresholds ( $c_{1}=0.4 \mathrm{~m} / \mathrm{s}^{2}$, and $c_{2}=0.8 \mathrm{~m} / \mathrm{s}^{2}$ in dashed lines) set 
TABLE I

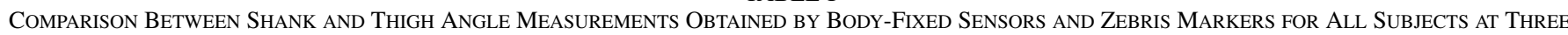

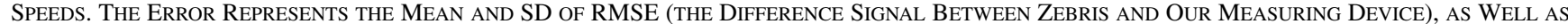
the Mean and SD of the Correlation CoefFicient Between the Two Measuring Systems

\begin{tabular}{lcccccc}
\hline \multirow{2}{*}{ Segment } & \multicolumn{2}{c}{ Slow $(2 \mathrm{~km} / \mathrm{h})$} & \multicolumn{2}{c}{ Intermediate $(3 \mathrm{~km} / \mathrm{h})$} & \multicolumn{2}{c}{ Fast $(4 \mathrm{~km} / \mathrm{h})$} \\
& RMS Error & Correlation Coeff. & RMS Error & Correlation Coeff. & RMS Error & Correlation Coeff. \\
& & & & & & \\
\hline Shank & $0.74 \pm 0.18$ & $0.9991 \pm 0.0008$ & $0.73 \pm 0.14$ & $0.9994 \pm 0.0004$ & $0.78 \pm 0.17$ & $0.9994 \pm 0.0003$ \\
Thigh & $1.42 \pm 0.23$ & $0.9985 \pm 0.0002$ & $1.57 \pm 0.35$ & $0.9986 \pm 0.0003$ & $1.69 \pm 0.48$ & $0.9986 \pm 0.0008$ \\
\hline
\end{tabular}

for hysteresis thresholding. The result of hysteresis thresholding is the binary mask $M_{1}$ [Fig. 6(b)]. The second step applies a morphological opening filter on $M_{1}$ to eliminate narrow pulses shorter than $T_{0}$ [Fig. 6(c)].

Fig. 7 shows how the two angle information obtained by gyroscope $\left(\phi_{g}\right)$ and virtual accelerometer $\left(\phi_{a}\right)$ are combined to estimate the offset and drift $d(t)$. The process consists of subtracting the two signals using (11) followed by applying the piecewise cubic hermite interpolation. The estimated drift $d(t)$ is then used to calculate the correct orientation of shank $\left(\phi_{\text {Shank }}\right)$ using (12).

The comparison between the reference angles and the measured angles using the proposed method for shank motion is shown in Fig. 8. The error $(\operatorname{Er}(t))$ is defined as the difference between the shank orientation angle $\left(\phi_{\text {Shank }}\right)$ estimated by the proposed method [Fig. 8(a)], and calculated from position data as measured by the reference system [Fig. 8(b)]. It can be seen that the value of the $\operatorname{Er}(t)$ [Fig. 8(c)] is very small $\left(<1.2^{\circ}\right)$. Similarly, the thigh orientation angle $\left(\phi_{\text {Thigh }}\right)$, calculated using (13), is compared with its corresponding reference angle as shown in Fig. 9.

The whole results of the validating shank and thigh angles with reference system are summarized in Table I, which outlines the mean and standard deviation of root-mean-square errors (RMSEs) and correlation coefficients of all subjects. The average RMSE for shank angles was $1.0^{\circ}$ (mean $=0.5^{\circ}$, $\mathrm{SD}=0.8^{\circ}$ ) and the average correlation coefficient was 0.999 . In the same way, the average RMSE for thigh angles was $1.6^{\circ}$ (mean $\left.=0.1, \mathrm{SD}=1.4^{\circ}\right)$ and the average correlation coefficient was 0.998 .

\section{B. Visualization}

Our system was tested on actual patients at the University Hospital (CHUV-HOSR). Because curves such as those produced by our system are not that easy to interpret even by physicians who are well trained in its use, we have developed the tool depicted by Fig. 10. It visualizes the outcome of clinical protocols and provides useful information for functional assessment of patients with hip or knee arthroplasty.

Given the absolute shank and thigh angles, we can produce animations such as the one Fig. 10. The lower-limb segments are modeled as a kinematic chain of articulated rigid links, whose position is specified by a set of angles. Our model contains 6 joints-hips, knees and ankles. The animation software takes data in the form of 2-D sagittal orientation angles, transforms them into quaternions [27], and computes the skeleton's configuration and position at each frame.
The tool gives the physician visually appealing and easy to interpret information about how the patient performs several activities such as walking at different speeds or climbing ramps and stairs. Because the animated skeleton is 3-D it can be viewed from arbitrary angles, thereby further helping the physician to interpret the results. In addition, the visualization tool's interface lets us evaluate the progression of the same patient over time by superposing several skeletons, as shown in the rightmost window of Fig. 10. To this end, a time-normalization of gait cycles was performed by detecting heel strike instances [22] before superposing the two cycles. The black skeleton corresponds to the baseline and the gray one the patient's condition $6 \mathrm{mo}$ after surgery. This tool allows for better evaluation of the patient's medical needs after surgery and is already used by CHUV-HOSR clinicians.

\section{DisCUSSION AND CONCLUSION}

The proposed method based on body-fixed sensors gave an accurate estimation of lower limbs orientations during gait. The results of all tests (Table I) were very close to those of the reference system presenting small errors in RMS, mean, and standard deviation of the difference signal, reflecting accurate and precise estimates respectively; and excellent correlation coefficients reflecting highly linear response.

Our method compares favorably with other methods used to estimate shank or body segment orientation. Mayagoitia et al. [28] showed that shank and thigh inclination angles can be measured with the need of signal integration with eight accelerometers as wells as two gyroscopes fixed on two rigid metal plate. They found that RMSE for shank ranges from $1.3^{\circ}$ to $2.7^{\circ}$. Using single gyroscope on shank, Tong and Granat [9] estimated the RMSE of relative shank angle (not absolute) to around $4.95^{\circ}$ while using two gyroscopes (shank and thigh) we had previously found for arthritis patients standard errors of $3.3^{\circ}$ and $4.2^{\circ}$, respectively, for relative shank and thigh orientation [10]. In comparison with the methods using Kalman filtering, the proposed method has faster response, no phase delay, no convergence problem, and less computational load. Although the Kalman filter can have a higher accuracy in many applications, and can even be applied in real time, the performance of the filter will considerably be reduced in measuring orientation angle of segments, like shank, with fast movements and large centripetal acceleration components [5], [7]. Many authors designed Kalman filters to fuse gyroscope, accelerometer and/or magnetometer signals [5]-[7], [12]-[15]. However, the performance of the filter was only validated on the motions of segments such as trunk or head, which have relatively slower motions than shank and thigh. Nevertheless, 


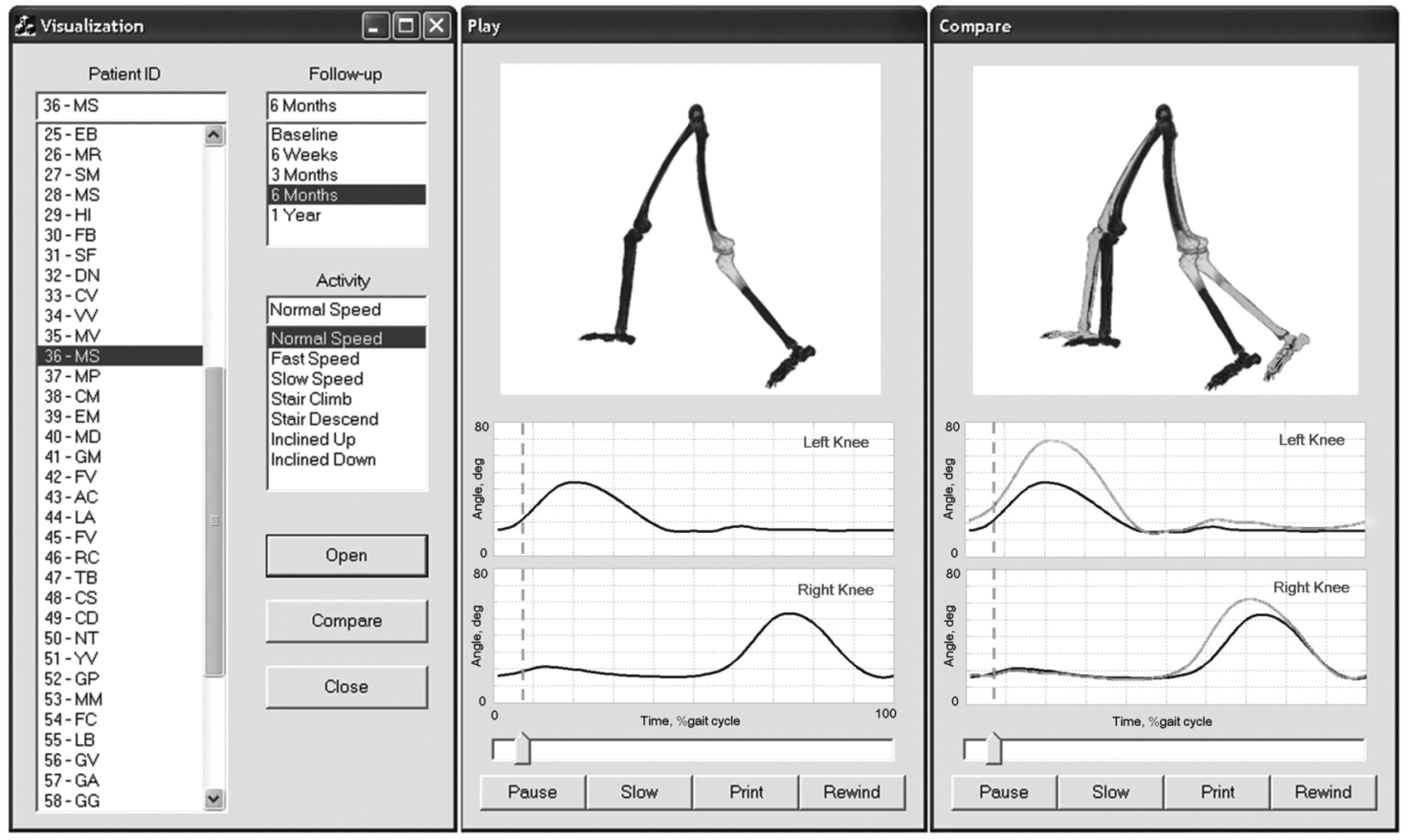

Fig. 10. Interface of our visualization tool. The animated skeleton of a patient during walking with knee arthroplasty is shown in the middle window. The knee prosthesis is colored in light gray. The right window compares the gait of the same patient just before operation (black) with 6 mo later (gray).

the presented algorithm in this paper is limited to postprocessing of data, and the time lag to find low acceleration points is an inevitable consequence.

In contrast to the method presented in [1] for measuring joint angle, the proposed method in this paper requires to find low acceleration points in order to measure orientation of a segment with respect to a fixed frame. Joint angle is the relative angle of two connecting segments with a known center of rotation at the joint point. So, a pair of virtual sensors could be placed at the joint center of rotation to find joint angle [1]. A similar concept was used in this paper to find orientation of a segment by considering it as the joint angle between the segment and a fixed frame. However, a freely moving segment has not a constant center of rotation. So only at zero motion points the assumption is valid as the center of rotation is known.

From a practical standpoint, misalignment of the sensors or sensor deviation during movement reduces the system's accuracy, such that the sensor reading is multiplied by the cosine of the misalignment angle $\epsilon$. However, this will not seriously disturb the signals, since $\cos \epsilon \approx 1$ if $\epsilon$ is sufficiently small. The accuracy of the angles are still limited by the bias drift of the accelerometers, however it is much less severe than integrating gyro drift. In addition, skin motion artifact, a common source of error to all body mounted devices, affects the measurement accuracy. The thigh sensor is more susceptible to skin and soft tissue artifact where the majority of the femur is concealed by a substantial amount of soft tissue [29]-[31]. The effect of skin artifact was minimized by using adequate elastic band to fix the sensors, and applying low-pass filtering on the raw signal.

The results show that using gait constraints imposed by ankle joint, and virtually placing an accelerometer on ankle, can improve the accuracy of the measurements. In addition, the information of the virtual sensor on ankle can further be used to de- tect foot-flat phases. Identifying the foot-flat phase (loading response) of the gait cycle is one of the most demanding tasks in the study of human locomotion [32], [33].

The resulting ambulatory system is light ( $300 \mathrm{gr})$ and easily mountable, which allows long-term monitoring without hindrance to the natural gait [22]. The gait visualization tool for lower limbs angle tracking gives the physician an intuitive information about how a patient performs several activities. This visual information has proved very useful in an actual hospital setting to help physicians interpret the kinematic curves.

The current model is limited to 2-D sagittal measurement of lower limbs. However, in gait analysis, a 2-D sagittal approach seems to be satisfactory, because sagittal plane is the plane where majority of the movement takes place, and gives a lot of information for gait pathologies [9]. Therefore, many applications in gait analysis and orthopedics are concerned with the proposed method. For example, lower limbs absolute angles can be used to provide outcome evaluation after orthopedic surgery since there is a correlation between functional improvement found by clinical Harris hip scores (HHSs) [34] and range of flexion of the thigh $(r=0.69$ and $p<0.01)$ in arthritis patients [35]. Moreover, main activity such as lying, sitting, standing, walking, and stair climbing can be identified by a subtle combination of the shank, thigh, and knee angle in sagittal plane [33], [36] and provide in this way useful outcome for mobility improvement after hip arthroplasty.

However, the approach could be extended to frontal plane analysis to obtain a quasi-3-D reconstruction by employing 3-D gyroscopes and accelerometers on each site. Furthermore, we are also working on recovering motion in the frontal plane by combining the information provided by body-fixed sensors with image-data from inexpensive and commercially available synchronized cameras [37], [38]. This extended approach should 
let us incorporate full 3-D motion into gait analysis and will be the subject of future work.

\section{ACKNOWLEDGMENT}

The authors are grateful to J. Gramiger and P. Morel in the design of the ambulatory system; and C. Voracek, C. Pichonnaz, and A. Poloni for their assistance in collecting the data.

\section{REFERENCES}

[1] H. Dejnabadi, B. M. Jolles, and K. Aminian, "A new approach to accurate measurement of uniaxial joint angles based on a combination of accelerometers and gyroscopes," IEEE Trans. Biomed. Eng., vol. 52, no. 8, pp. 1478-1484, Aug. 2005.

[2] K. Meyer, H. L. Applewhite, and F. A. Biocca, "A survey of position trackers," Presence: Teleoperators and Virtual Environments, vol. 1, pp. 173-200, Spring, 1992.

[3] M. Saleh and G. Murdoch, "In defense of gait analysis-observation and measurement in gait assessment," J. Bone Joint Surg. -Br. Vol., vol. 67, pp. $237-241,1985$.

[4] A. E. Patla and S. D. Clous, "Visual assessment of human gait: reliability and validity," Rehabil. Res., vol. 1, pp. 87-96, 1997.

[5] H. J. Luinge and P. H. Veltink, "Inclination measurement of human movement using a 3-D accelerometer with autocalibration," IEEE Trans. Neural Syst. Rehabil. Eng., vol. 12, no. 1, pp. 112-121, Mar. 2004.

[6] E. Foxlin, "Inertial head-tracker sensor fusion by a complementary separate-bias Kalman filter," presented at the 1996 IEEE Virtual Reality Ann. Int. Symp., 1996.

[7] H. Rehbinder and X. Hu, "Drift-free attitude estimation for accelerated rigid bodies," Automatica, vol. 40, pp. 653-659, 2004.

[8] J. R. W. Morris, "Accelerometry-a technique for the measurement of human body movements," J. Biomech., vol. 6, pp. 729-732, 1973.

[9] K. Tong and M. H. Granat, "A practical gait analysis system using gyroscopes," Med. Eng. Phys., vol. 21, pp. 87-94, 1999.

[10] K. Aminian, C. Trevisan, B. Najafi, H. Dejnabadi, C. Frigo, E. Pavan, A. Telonio, F. Cerati, E. C. Marinoni, P. Robert, and P.-F. Leyvraz, "Evaluation of an ambulatory system for gait analysis in hip osteoarthritis and after total hip replacement," Gait Posture, vol. 20, pp. 102-107, 2004.

[11] A. Heyn, R. E. Mayagoitia, A. V. Nene, and P. H. Veltink, "The kinematics of the swing phase obtained from accelerometer and gyroscope measurements," presented at the 18th Annu. Int. Conf. IEEE Engineering in Medicine and Biology Society—Bridging Disciplines for Biomedicine, 1996

[12] R. Zhu and Z. Zhou, "A real-time articulated human motion tracking using tri-axis inertial/magnetic sensors package," IEEE Trans. Neural Syst. Rehabil. Eng., vol. 12, no. 2, pp. 295-302, Jun. 2004.

[13] B. Barshan and H. F. Durrant-Whyte, "Inertial navigation systems for mobile robots," IEEE Trans. Robot. Automat., vol. 11, no. 3, pp. 328-342, Jun. 1995.

[14] J. L. Marins, X. Yun, E. R. Bachmann, R. B. McGhee, and M. J. Zyda, "An extended Kalman filter for quaternion-based orientation estimation using MARG sensors," presented at the 2001 IEEE/RSJ Int. Conf. Intelligent Robots and Systems, 2001.

[15] H. J. Luinge and P. H. Veltink, "Measuring orientation of human body segments using miniature gyroscopes and accelerometers," Med. Biol. Eng. Comput., vol. 43, pp. 273-282, 2005.

[16] C. V. C. Bouten, K. T. M. Koekkoek, M. Verduin, R. Kodde, and J. D. Janssen, "A triaxial accelerometer and portable data processing unit for the assessment of daily physical activity," IEEE Trans. Biomed. Eng., vol. 44, no. 3, pp. 136-147, Mar. 1997.

[17] B. Kemp, A. J. M. W. Janssen, and B. van der Kamp, "Body position can be monitored in 3D using miniature accelerometers and earthmagnetic field sensors," Electroencephalogr. Clin. Neurophysiol./Electromyogr. Motor Contr., vol. 109, pp. 484-488, 1998.

[18] E. Foxlin and M. Harrington, "WearTrack: a self-referenced head and hand tracker for wearable computers and portable VR," presented at the 4th Int. Symp. Wearable Computers 2000., 2000.

[19] J. Canny, "A computational approach to edge-detection," IEEE Trans. Pattern Anal. Mach. Intell., vol. PAMI-8, pp. 679-698, 1986.
[20] C. Ronse and H. J. A. M. Heijmans, "The algebraic basis of mathematical morphology: II. Openings and closings," CVGIP: Image Understanding, vol. 54, pp. 74-97, 1991.

[21] F. N. Fritsch and R. E. Carlson, "Monotone piecewise cubic interpolation," SIAM J. Numerical Anal., vol. 17, pp. 238-246, 1980.

[22] K. Aminian, B. Najafi, C. Bula, P.-F. Leyvraz, and P. Robert, "Spatiotemporal parameters of gait measured by an ambulatory system using miniature gyroscopes," J. Biomech., vol. 35, pp. 689-699, 2002.

[23] R. M. Kiss, L. Kocsis, and Z. Knoll, "Joint kinematics and spatialtemporal parameters of gait measured by an ultrasound-based system," Med. Eng. Phys., vol. 26, pp. 611-620, 2004.

[24] Z. M. GmbH, "Measuring system for 3D-motion analysis CMS-HS," Tech. Data and Operating Instructions Text Release, vol. 8/99, 1999.

[25] H. M. Overhoff, D. Lazovic, M. Liebing, and C. Macher, "Total knee arthroplasty: coordinate system definition and planning based on 3-D ultrasound image volumes," in Proc. 15th Int. Congr. Exhibit. Computer Assisted Radiology and Surgery (CARS), 2001, vol. 1230, pp. 292-299.

[26] A. Savitzky and M. J. E. Golay, "Smoothing + differentiation of data by simplified least squares procedures," Analytical Chem., vol. 36, p. $1627,1964$.

[27] K. Shoemake, "Animating rotations with quaternion curves," in Proc. Computer Graphics (SIGGRAPH '85), 1985, vol. 19, pp. 245-254.

[28] R. E. Mayagoitia, A. V. Nene, and P. H. Veltink, "Accelerometer and rate gyroscope measurement of kinematics: an inexpensive alternative to optical motion analysis systems," J. Biomech., vol. 35, pp. 537-542, 2002.

[29] A. Cappozzo, F. Catani, A. Leardini, M. Benedetti, and U. D. Croce, "Position and orientation in space of bones during movement: experimental artefacts," Clin. Biomech., vol. 11, pp. 90-100, 1996.

[30] C. Reinschmidt, A. J. van den Bogert, A. Lundberg, B. M. Nigg, N. Murphy, A. Stacoff, and A. Stano, "Tibiofemoral and tibiocalcaneal motion during walking: external vs. skeletal markers," Gait Posture, vol. 6, pp. 98-109, 1997.

[31] C. Reinschmidt, A. J. van den Bogert, B. M. Nigg, A. Lundberg, and N. Murphy, "Effect of skin movement on the analysis of skeletal knee joint motion during running," J. Biomech., vol. 30, pp. 729-732, 1997.

[32] A. E. Hunt, R. M. Smith, M. Torode, and A.-M. Keenan, "Inter-segment foot motion and ground reaction forces over the stance phase of walking," Clin. Biomech., vol. 16, pp. 592-600, 2001.

[33] B. Coley, B. Najafi, A. Paraschiv-Ionescu, and K. Aminian, "Stair climbing detection during daily physical activity using a miniature gyroscope," Gait Posture, vol. 22, pp. 287-294, 2005.

[34] W. H. Harris, "Traumatic arthritis of hip after dislocation and acetabular fractures - treatment by mold arthroplasty - an end-result study using a new method of result evaluation," J. Bone Joint Surg.-Am. Vol., vol. A 51, p. 737, 1969.

[35] H. Dejnabadi, B. M. Jolles, B. Najafi, C. Trevisan, E. C. Marinoni, and K. Aminian, "A Robust Gait parameterization technique for hip arthroplasty outcome evaluation," in Proc. ISPGR, Posture and Gait Throughout the Lifespan, S. R. Lord and H. B. Menz, Eds., 2003, p. 52.

[36] M. Morlock, E. Schneider, A. Bluhm, M. Vollmer, G. Bergmann, V. Muller, and M. Honl, "Duration and frequency of every day activities in total hip patients," J. Biomech., vol. 34, pp. 873-881, 2001.

[37] E. Casanova, H. Dejnabadi, B. M. Jolles, K. Aminian, and P. Fua, "3D motion analysis and synthesis from inertial gyroscopes and image data," in Proc. ESMAC 2004, 2004, vol. 20S, p. S85.

[38] R. Plankers and P. Fua, "Articulated soft objects for multiview shape and motion capture," IEEE Trans. Pattern Anal. Mach. Intell., vol. 25, no. 9, pp. 1182-1187, Sep. 2003.

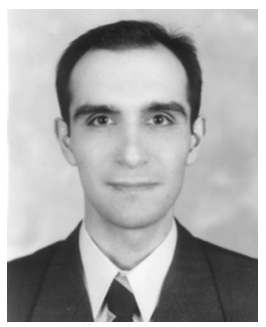

Hooman Dejnabadi received the B.Sc. and M.Sc. degrees in electrical engineering from Sharif University of Technology, Tehran, Iran, in 1993 and 1996 respectively. He is currently working towards the Ph.D. degree in biomedical engineering at the Laboratory of Movement Analysis and Measurement, Ecole Polytechnique Fédérale de Lausanne (EPFL), Lausanne, Switzerland.

He was with Fara Sanat Shomal Co. and RadRavesh Co. from 1993 to 2001 as a Research Engineer on NDT systems. His research interests include biomedical signal processing, ambulatory systems, and movement analysis. 


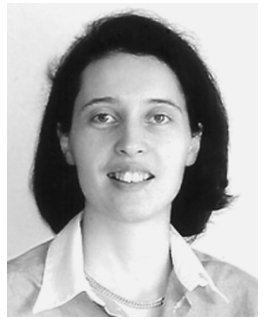

Brigitte M. Jolles received the MSc degree in microtechnology engineering from the Swiss Federal Institute of Technology (EPFL), Lausanne, Switzerland, in 1990, the M.D. degree from University of Lausanne (UNIL) in 1995, and the specialist degree in orthopedic surgery. She received the Diploma in Clinical Epidemiology from the University of Toronto (UofT), Toronto, ON, Canada, in 2002.

She is an Orthopaedic Surgeon, an Engineer with the EPFL, and Assistant Professor at the UNIL. In 2001-2002, she worked as a Clinical and Research Fellow in the Department of Orthopedic Surgery at UofT. She became Assistant Professor at UNIL in February 2005. She is part of the surgeon staff at the Orthopaedic Hospital of the University of Lausanne and teaches at the pregraduate and postgraduate levels at UNIL and other institutions. Her research interests include joint arthroplasty and ambulatory systems for movement analysis.

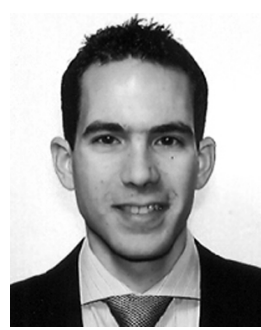

Emilio Casanova received the M.Sc. degree in microtechnology engineering from the Swiss Federal Institute of Technology (EPFL) in 2002. He received his postgraduate certificate in signal and image processing in 2002.

He was at with the Biomedical Imaging Group of M. Unser (BIG/EPFL) in 2002 as an R\&D Engineer. In January 2003, he joined the Computer Vision Laboratory (CVLAB/EPFL) as a Research Assistant. His research interests include image processing, stereovision, camera-based systems, and sensors fusion algo-

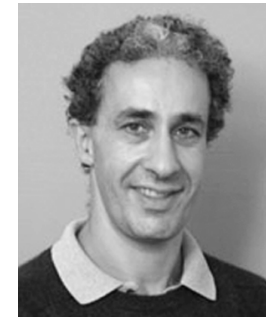

Pascal Fua (M'01) received a degree from Ecole Polytechnique, Paris, France, in 1984 and the Ph.D. degree in computer science from the University of Orsay, Orsay, France, in 1989.

$\mathrm{He}$ is now a Professor in the Swiss Federal Institute of Technology (EPFL) School of Computer and Communication Science. Before that, he worked at SRI International and at INRIA Sophia-Antipolis as a computer scientist. His research interests include human body modeling from images, optimization-based techniques for image analysis and synthesis, and the use of information theory in the area of model-based vision. He has (co)authored over 100 publications in refereed journals and conferences.

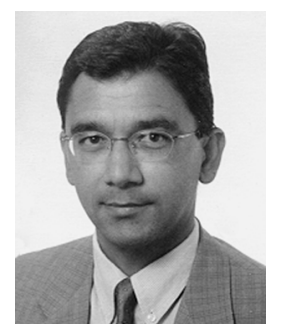

Kamiar Aminian (M'89) received the electrical engineering degree in 1982 and the Ph.D. degree in biomedical engineering in 1989 from Ecole Polytechnique Fédérale de Lausanne (EPFL), Lausanne, Switzerland.

He worked as a Research Associate in the Metrology Laboratory, EPFL and as an Assistant Professor at Sharif University of Technology, Tehran, Iran. In January 2002, he joined the School of Engineering of EPFL where he is the Head of the Laboratory of Movement Analysis and Measurement. He teaches in the areas of sensors and medical instrumentation. His research interests include transducers, movement analysis, ambulatory systems, and biomedical signal processing.

rithms. 